\title{
Numerical Study of Blood Flow through Symmetry and Non- Symmetric Stenosis Artery under Various Flow Rates
}

\author{
Mohammed Nasir Uddin ${ }^{1 *}$, M.A. Alim ${ }^{2}$ \\ ${ }^{1}$ Department of Information and Communication Technology (ICT), Bangladesh University of Professionals \\ (BUP),Bangladesh. \\ ${ }^{2}$ Department of Mathematics, Bangladesh University of Engineering \& Technology (BUET), Bangladesh
}

\begin{abstract}
This study investigated the significance of symmetry and non-symmetry stenosis effects of blood flow and quantifies some of the most relevant non Newtonian characteristics of blood flow in blood vessels. The models studied in this work are the Newtonian and Non-Newtonian (Oldroyd-B) models, as well as their generalized (shear-thinning) modifications. The governing system of equations is based on incompressible Navier-Stokes equations which are generalized to take into account viscoelasticity and shear-thinning properties of blood flow. Finite element method is used for the solution of the governing system of equations. Mathematical tests are performed on an idealized symmetric stenosis and a realistic stenosed carotid bifurcation reconstructed from medical images. Model sensitivity tests are achieved with respect to the characteristic flow rate to evaluate its impact on the observed non-Newtonian effects. The numerical simulation is performed for various flow rates 0.05 to $2 \mathrm{~cm}^{3} / \mathrm{s}$ at Reynold numbers, Re $=10^{2}$ and Weissenberg numbers, $W i=0.6$ with good convergence of the iterative scheme. Results from the blood flow simulations indicate that non-Newtonian behavior has considerable effects on instantaneous flow patterns. Different effects for these models are presented numerically.
\end{abstract}

Keywords: Blood flow, Oldroyd-B model, Finite element method, stenosis cavity, viscoelasticity, shearthinning

\section{Nomenclature \\ $\sigma \quad$ Stress Tensor $(\mathrm{Pa})$ \\ $\sigma_{v} \quad$ Viscous stress tensor $(\mathrm{Pa})$ \\ $\sigma_{n} \quad$ Solvent stress tensor $(\mathrm{Pa})$ \\ $L \quad$ height and the enclosure (m) \\ I Identity matrix \\ Wi Weissenberg number \\ $p$ pressure $\left(\mathrm{Nm}^{-2}\right)$ \\ $P \quad$ non-dimensional pressure \\ Re Reynold number \\ $q \quad$ Flow rate $\left(\mathrm{m}^{2} / \mathrm{s}\right)$ \\ $\lambda_{x} \quad$ Relaxation time \\ $\lambda_{d} \quad$ Retardation time \\ $V \quad$ Symmetry velocity gradient}

$\begin{array}{ll}\mu_{n} & \text { Polymer viscosity } \\ \mu_{s} & \text { Solvent viscosity } \\ V^{\prime} & \text { Anti-symmetry velocity gradient } \\ \dot{\gamma} & \text { Rate of deformation tensor } \\ u, v & \text { velocity components }\left(\mathrm{ms}^{-1}\right) \\ U, V & \text { Dimensionless velocity components } \\ & \text { Greek symbols } \\ \mu & \text { Viscosity } \\ \gamma & \text { Deformation tensor } \\ \rho & \text { density of the fluid }\left(\mathrm{kgm}^{-3}\right) \\ v & \text { kinematic viscosity of the fluid }\left(\mathrm{m}^{2} \mathrm{~s}^{-1}\right) \\ \tau_{\mathrm{s}} & \text { Extra-stress tensor }\end{array}$

I. Introduction

At the beginning of the discovery that the fatal cardiovascular disease arteriosclerosis or stenosis and aneurysm affects the flow of blood in the arteries and leads to serious circulatory disorders, this area of biomechanics has been receiving the attention of researchers during the recent decades [14]. At present time medical researchers, bioengineers and numerical scientists join efforts with the purpose of providing numerical simulations of human blood flow system in different conditions. In our blood circulation system, there are some strong hemodynamical features that can change in the rheological properties of blood and its components. It plays a major role in the development and progression of atherosclerotic plaques and other arterial lesions [18]. In any physiological and pathological situations, the numerical study is an important tool for the interpretation and analysis of the circulatory system and can capture the rheological response of blood over a range of physiological flow conditions accurately [7]. Stenosis developed in the arteries pertaining to brain can cause cerebral strokes and the one developed in the coronary arteries can cause myocardial infarction which leads to heart failure [8]. It has been reported that the fluid dynamical properties of blood flow through nonuniform cross section of the arteries play a major role in the fundamental understanding and treatment of many cardiovascular diseases [19]. The analysis of blood flow through tapered tubes is very important in understanding the behavior 
of the blood flow as the taper of the tube is a vital factor in the pressure development [9]. Thurston [17] was among the earliest to recognize the viscoelastic nature of blood and that the viscoelastic behavior is less prominent with increasing shear rate. Anand et.al. [1] have developed a model that is suitable for blood simulation and it contains Oldroyd-B fluid flow characteristics. Rajagopal and Srinivasa [13] have described a thermodynamic framework which is well suited for describing the viscoelastic response (blood) of bodies with multiple configurations. To captures the shear-thinning rheological behavior a DA method has developed by Elia et. al. [5] to show the numerical simulation of blood flow in 2D idealized stenosis with wall shear stress effect. Numerical simulation of generalized Newtonian and Oldroyd-B fluid have studied by V. Prokop et al. [10] with an extended computational domain. G. Telma et. al. [16] have studied the blood flow simulations can improved by integrating known data in 2D idealized stenosis vessels. The Numerical modelling of viscous and viscoelastic fluids flow through the branching channel have discussed by K. Radka et.al. [11]. Recently, M. Mahfoud et.al. [12] have shown that the pulsatile blood flow through an arterial stenosis to evaluate the flow characteristics and the wall shear stress under physiological conditions. In the present thesis, a numerical investigation of blood flow through stenosis and aneurysm have been studied. The governing mass, momentum and Oldroyd-B equation are expressed in a normalized primitive variables formulation. In this thesis, a finite element method for steady-state incompressible blood flows has been developed.

\section{Model Specifications}

The physical model considered here is shown in Fig.1, along with the important geometric parameters. The stenosed vessel is assumed to be two-dimensional with diameter $D=2 R=6.2 \mathrm{~mm}$ which reduces smoothly to one half in the stenosed region. The parabolic velocity profile is prescribed at the inlet and pressure is fixed to a constant at outlet. On the walls, no-slip conditions are used for velocity and homogeneous Neumann condition for the pressure. For Oldroyd-B model variables $\left(\sigma_{v}\right)$ there is zero kept at the inlet and homogeneous Neumann condition at the walls and at the outlet. The stenosis cross-sectional area ration is $2: 1$ and thus a significant local acceleration of the flow is expected.

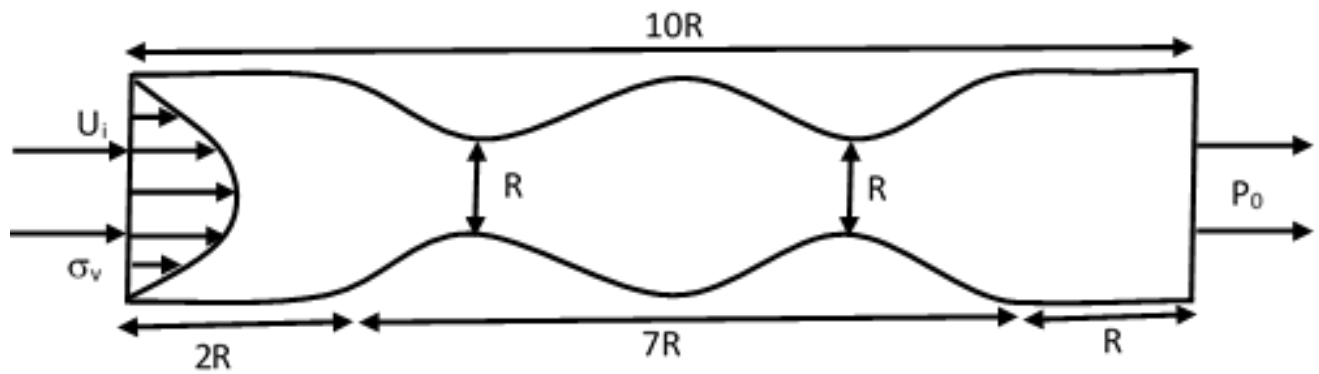

Figure-1. Schematic diagram of the physical system

\subsection{Mathematical Model}

The fundamental laws used to solve for the variables velocity, pressure and shear stress, provided the viscosity function, and flow parameters. With respect to boundary conditions the problems are the conservation of mass (continuity equations), conservation of momentums (momentum equations), and Oldroyd-B (viscoelasticity equations), which constitute a set of coupled, nonlinear, and partial differential equations. To obtain a system of dimensionless variables, we use some scaling properties of the system to introduce Reynolds number $(R e)$ and Weissenberg number $(W i)$ that measures the effect of viscosity and elasticity on blood flow for steady state.

Using non-dimensional variables defined in the nomenclature, the non-dimensional governing equations (1), (2) and (3) are obtained for 2D stenosis vessel in domain $\Omega$ as follows:

\section{Continuity Equation}

$$
\nabla \cdot \mathbf{U}=0
$$

Momentum Equations

$$
\operatorname{Re}[(\mathbf{U} . \nabla) \mathbf{U}]=-\nabla \mathbf{P}+(1-\lambda) \Delta \mathbf{U}+\nabla \cdot \boldsymbol{\sigma}+\mathbf{f}
$$

Oldroyd-B constitutive equation:

$$
\mathrm{W}_{\mathrm{i}}[(\mathbf{U} . \nabla) \boldsymbol{\sigma}]+\boldsymbol{\sigma}=2 \mu_{\mathrm{v}} \mathbf{V}(\mathbf{U})+\mathrm{W}_{\mathrm{i}}\left[(\nabla \mathbf{U}) \boldsymbol{\sigma}+\boldsymbol{\sigma}(\nabla \mathbf{U})^{\mathrm{t}}\right]
$$




\subsection{Boundary conditions}

The boundary conditions are

At inlet:

(i) Dirichlet boundary conditions for velocity vector are used on the boundary $\partial \Omega$.

(ii) For a pressure and the stress tensor Neumann boundary condition is used on the boundary $\partial \Omega$.

(iii) The developed parabolic velocity profile and the corresponding extra stresses components

$$
\begin{aligned}
& \mathrm{u}=1.5\left(1-\mathrm{s}^{2}\right), \mathrm{v}=0 \\
& \sigma_{11}=2 \mu_{\mathrm{v}} \mathrm{Wi}\left(\frac{\partial \mathrm{u}}{\partial \mathrm{y}}\right)^{2} \\
& \sigma_{12}=\mu_{\mathrm{v}} \frac{\partial \mathrm{u}}{\partial \mathrm{y}} \\
& \sigma_{22}=0
\end{aligned}
$$

At outlet:

(i) At outflow boundary pressure value is constant and for the velocity vector and the stress tensor Neumann boundary condition is used.

(ii) Due to pressure force $\left(\mathrm{P}_{\mathrm{o}}\right)$ the stress is acting at the boundary

$$
\text { T. } \mathbf{n}=-\mathrm{P}_{\mathrm{o}} \mathbf{n}
$$

\section{At Boundary Wall:}

(i) On the walls, no slip conditions are used for the velocity together with the condition for the normal component of the extra stress: $\mathbf{u}=0$ and (T. $\mathbf{n}) . \mathbf{n}=0$ Where $\mathrm{n}$ is the boundary unit normal vector.

(ii) Homogenous Neumann boundary conditions are used for the pressure.

The above equations were normalized using the following dimensionless scales:

$\mathrm{x}=\mathrm{LX}, \mathrm{t}=\mathrm{Lt}^{*} / \mathrm{U}, \mathrm{u}=\mathrm{UU}_{\mathrm{o}}, \mathbf{v}=\mathrm{VU}_{\mathrm{o}}, \mathrm{p}=\mu \mathrm{UP} / \mathrm{L}, \mathrm{T}=\mathrm{U}_{\mu} \mathrm{T}^{*} / \mathrm{L}, \mathrm{f}=\mathrm{f}^{*} \mu \mathrm{U} / \mathrm{L}^{2}, \nabla=\nabla^{*} / \mathrm{L}, \mathrm{Wi}=\lambda_{\mathrm{x}} \mathrm{U} / \mathrm{L}, \mathrm{Re}=\rho \mathrm{UL} / \mu$ Reynolds number $(R e)$ and Weissenberg number $(W i)$ are dimensionless numbers. Small values of $W i$ mean that the fluid is little elastic and small values of $R e$ means that the fluid is very viscous.

\section{Numerical Analysis}

The governing equations along with the boundary conditions are solved numerically by employing Galergkin weighted residual finite techniques, in particular the commercial high-level finite element package COMSOL Multiphysics [2] and MATLAB Programming [6]. The application of this technique is well described by Taylor and Hood [15] and Dechaumphai [3,4]. The finite element formulation and computational procedure have been omitted here for brevity.

\section{Grid Independence Test}

Preliminary results are obtained to inspect the field variables grid independency solutions. Test for the accuracy of grid fineness has been carried out to find out the optimum grid number. In order to obtain grid independent solution, a grid refinement study is performed for a stenosis cavity with $\mathrm{Re}=10^{2}$ and $\mathrm{Wi}=0.6$ with blood flow rate $0.2 \mathrm{~cm}^{3} / \mathrm{s}$. Figure 3.3 shows the convergence of the average velocity (U) along the vessel axis with grid refinement. It is observed that grid independence is achieved with 22585 elements where there is insignificant change in velocity with further increase of mesh elements. Six different non-uniform grids with the following number of nodes and elements were considered for the grid refinement tests: 28442 nodes, 7355 elements; 50346 nodes, 9500 elements; 64240 nodes, 12586 elements; 69887 nodes, 14371 elements; 92573 nodes, 22585 elements, 98388 nodes, 29686 elements. From these values, 92573 nodes, 22585 elements can be chosen throughout the simulation to optimize the relation between the accuracy required and the computing time.

Table 1: Grid Sensitivity Check at $R e=100, \mathrm{Wi}=0.6$ and $\mathrm{q}=0.2 \mathrm{~cm}^{3} / \mathrm{s}$

\begin{tabular}{|l|l|l|l|l|l|l|}
\hline $\begin{array}{l}\text { Nodes } \\
\text { (elements) }\end{array}$ & 28442 & $\begin{array}{l}60346 \\
(7355)\end{array}$ & $\begin{array}{l}64240 \\
(12586)\end{array}$ & $\begin{array}{l}69887 \\
(14371)\end{array}$ & $\begin{array}{l}92573 \\
(22585)\end{array}$ & $\begin{array}{l}98388 \\
(29686)\end{array}$ \\
\hline$U$ & 0.14451252 & 0.14492982 & 0.14495304 & 0.14495446 & 0.14495578 & 0.14495579 \\
\hline Time (s) & 255.6 & 283.43 & 301.51 & 332.25 & 551.61 & 1007.65 \\
\hline
\end{tabular}




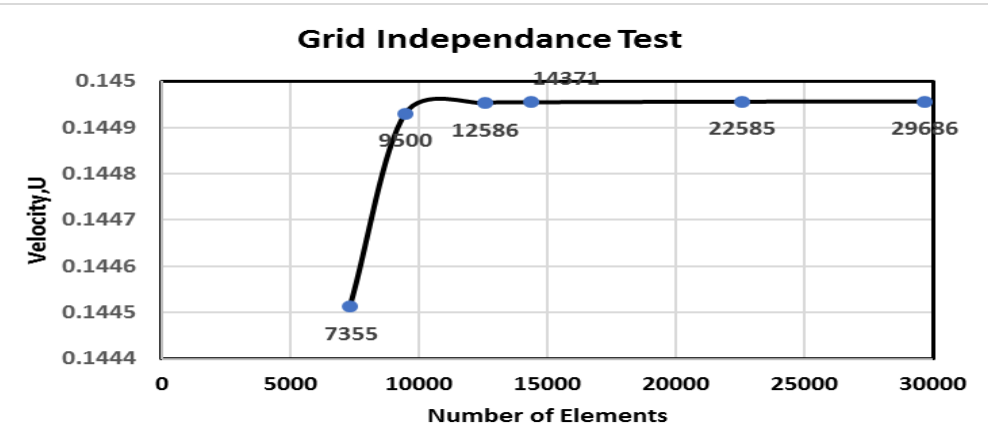

Figure-2: Convergence of average velocity with grid refinement for $R e=10^{2}$ and $\mathrm{Wi}=0.6$ with blood flow rate $0.2 \mathrm{~cm}^{3} / \mathrm{s}$

\section{Result and Discussion}

The objective of the present mathematical model is to understand and bring out the effects of symmetric stenosis, non-symmetric stenosis, wall shear stenosis, dimensionless numbers and various flow rate on blood flow for the Newtonian (N), generalized Newtonian (GN), Oldroyd-B (OD) and generalized OldroydB (GD) models. We have used the models mentioned in above to investigate the influence of the shear-thinning and viscoelastic effects on the behavior of blood in different flow situations. The solution of the Navier-Stoke equations is obtained under the same conditions as the solutions of the non-Newtonian equations. To show the non-Newtonian effects is used the comparison of flow field. We assume that the only change of blood viscosity $\mu_{\mathrm{n}}$ and extra stress $\mathrm{T}_{\mathrm{v}}$ for developed all four models. Numerical simulations and investigation have performed and compared for all four models. The following parameters [33] have been used for blood flow simulations in aorta

$$
\begin{array}{llll}
\mu_{0}=0.16 \text { Pa.s } & \mu_{\mathrm{n}}=0.0036 \text { Pa.s } \quad \mathrm{a}=1.23 & \mathrm{~b}=0.64 & \lambda=8.2 \mathrm{~s} \\
\rho=1050 \text { kg.m-3 } & \mathrm{L}_{\mathrm{w}}=0.003 \mathrm{~m} & \mathrm{~L}=0.03 \mathrm{~m} &
\end{array}
$$

In order to code validation, the blood flow simulation with above mentioned parameters for Newtonian model have solved, and the results have compared with those reported by V. Prokop et al. [42], obtained with an extended computational domain. A comparison between the simulation of velocity field is presented. The results from the present experiment are almost same as V. Prokop et al.

V. Prokop et al. (2013)

\section{Present Work}

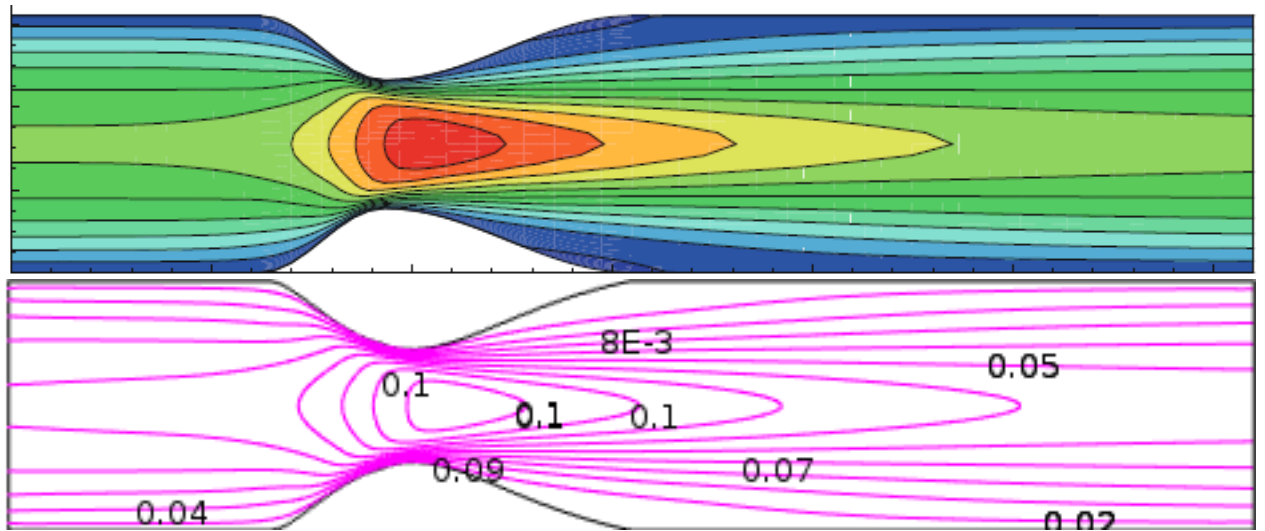

Figure 3: Comparison of velocity contour of blood flow through stenosis artery

\subsection{Symmetric Stenosis Effects on blood flow field}

The axial velocity contours of the four models Newtonian, Generalized Newtonian, Oldroyd-B, Generalized Oldroyd-B are presented in fig. 4 with the flow rate $\mathrm{q}=0.2 \mathrm{~cm}^{3} / \mathrm{s}$. The velocity profile has a significant changed on blood flow at throat of stenosis. Just behind the stenosis the reversal flow regions and flow separation are found with respect to the centerline. It is interesting to note that there are some permanent recirculation zone or flow separation zone formed at the throat of stenosis for four models. These recirculation zones are indicative of regions where the flow is reversed over a significant portion of each model. The recirculation zone becomes little shorter for generalized Newtonian and Oldroyd-B model. It is easy to comprehend, in the Newtonian case the characteristic blood viscosity is $\mu_{\mathrm{n}}$ and thus a shear-thinning viscosity leads to the increase of the local viscosity in the low-shear regions. Different results are found to choices of the other characteristic viscosity for the Newtonian model. The axial velocity profiles are shown in fig. 5 for all four 
models at dimensionless number $\mathrm{Wi}=0.6$ and $\mathrm{Re}=100$. Form the fig. 5 , the minimum value of velocity is found in $1^{\text {st }}$ constriction regions. It is clearly visible that the main effect of the blood shear-thinning behavior is noticeable in the recirculation zone, where the resistance to flow (local viscosity) increases substantially. The effects of viscoelasticity are about one order of magnitude lower in this case. For pulsatile flow or other flow rates or geometries, the viscoelastic effects may become more vital.

Newtonian

Generalized Newtonian

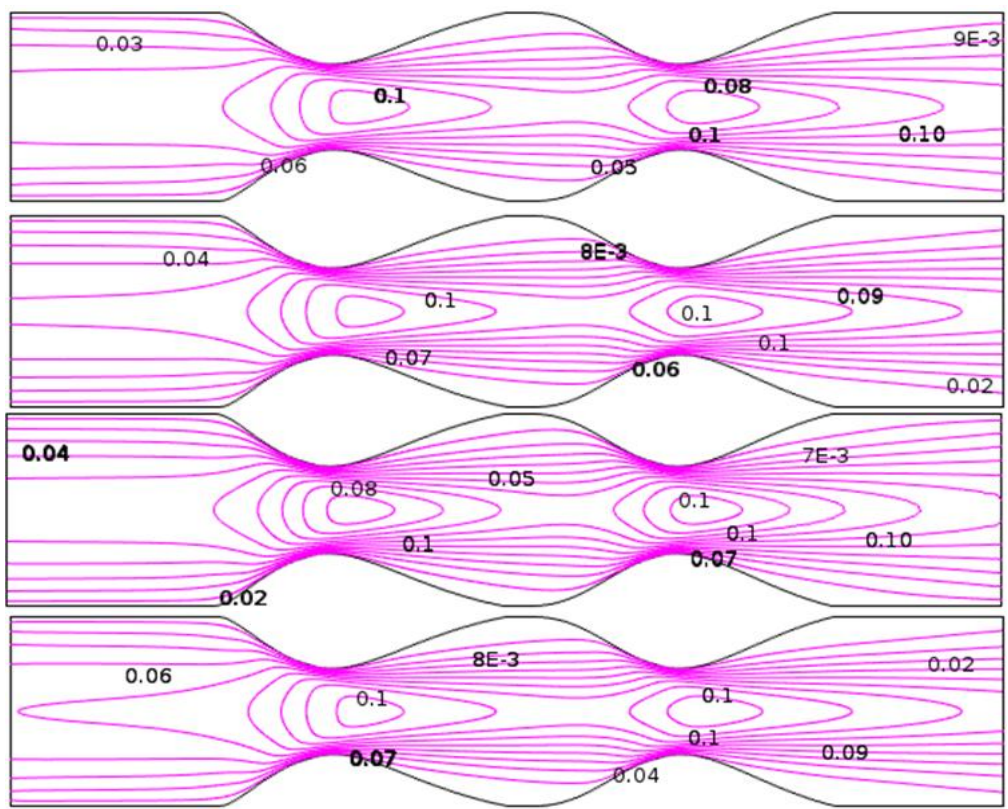

Figure 4: Velocity distribution on blood flow through Symmetric Stenosis at $R e=100$ and $W i=0.6$

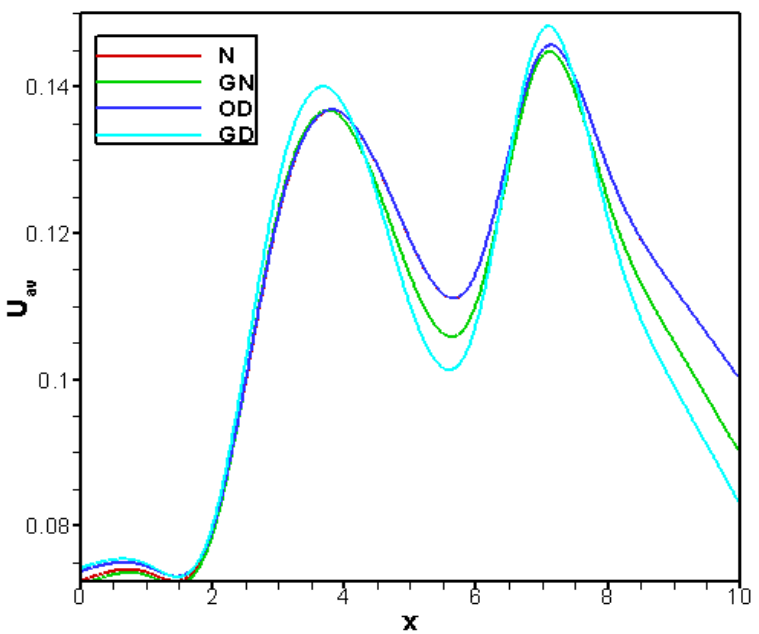

Figure 5: Effect of velocity profile of blood flow along vessel axis

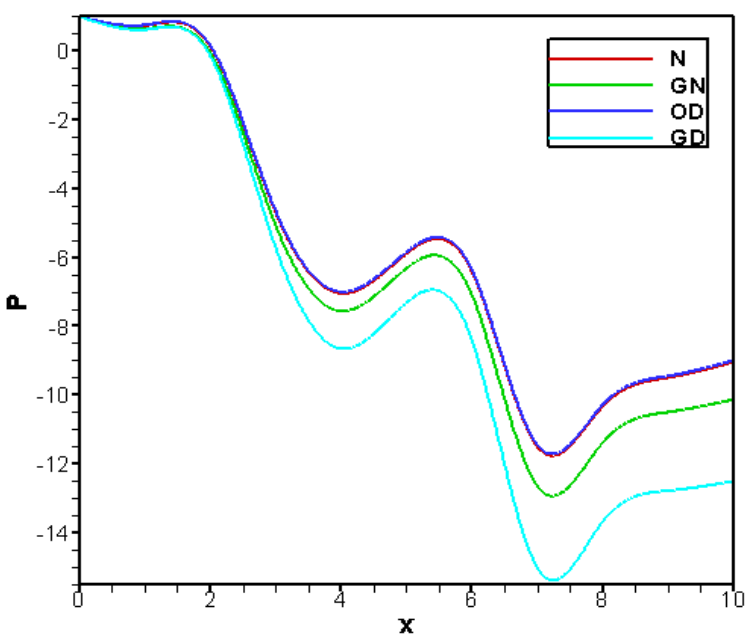

Figure 6: Effect of pressure profile of blood flow along vessel axis

\subsection{Symmetric Stenosis Effects on Pressure distribution}

In blood flow experiments blood pressure can easily be measured, in particular the pressure drop (i.e. the inlet-outlet pressure difference) needed to achieve a prescribed flow rate. In fig. 7, the axial pressure contours are presented for all the four models Newtonian, Generalized Newtonian, Oldroyd-B, Generalized Oldroyd-B with the flow rate $q=0.2 \mathrm{~cm}^{3} / \mathrm{s}$. These figures clearly show similarities among the four models at the inflow and outflow (far from the stenosis) of the artery with a developed pressure profile. While the difference in axial pressure profiles are pronounced at the around of stenosis, with some departures from the parabolic profile at second stenosis, due to great shear acting on the fluid in these regions. The pressure contour obtained relatively steep far the constriction regions but very intensive pressure gradient at stenosis.

Pressure is more dominated at stenosis regions because of the shear-thinning behavior of blood viscosity. It implies that, the flow is quicker than the non-Newtonian ones and its patterns remain in a disturbed state compare to others. The shear-thinning viscosity function leads to the growth of the local viscosity in the 
low-shear regions at the Newtonian case. The axial pressure profiles are shown in fig. 6 for all four models at dimensionless number $W i=0.6$ and $R e=100$. Form the fig. 6 , the lowest value of pressure is found in second stenosis region. The main effect of the blood shear-thinning behavior is visible in the throat of stenosis, where the local viscosity increases greatly. In fig. 6, the more negative values are found in the second stenosis region for generalized Newtoninan and Oldroyd-B model which leads to non-Newtonian fluid is slower than Newtonian fluid.

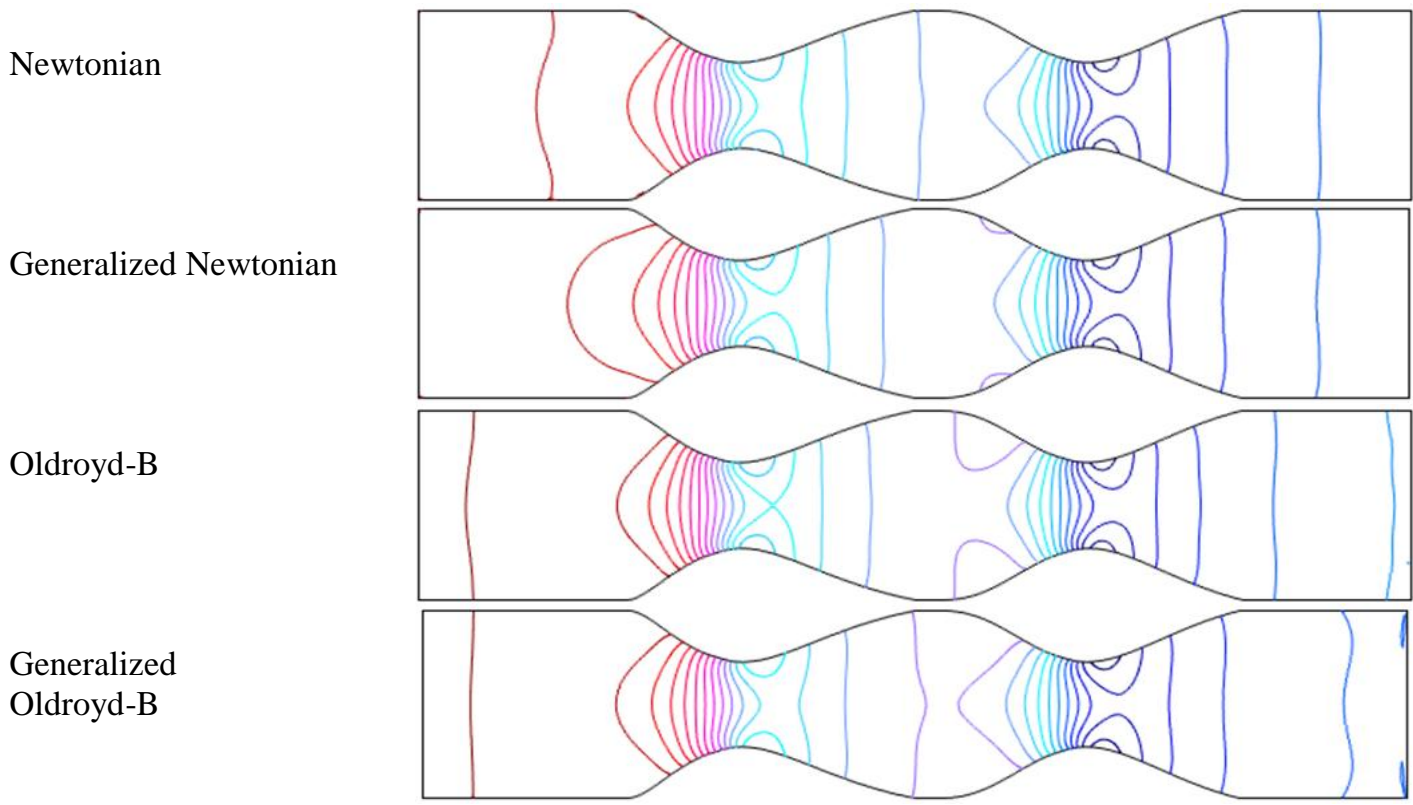

Figure 7: Pressure distribution on blood flow through Symmetric Stenosis at $R e=100$ and $W i=0.6$

\subsection{Non-symmetric Stenosis effect}

It is another remarkable effect on blood flow if the height of stenosis is different for velocity pressure distribution of the four dissimilar models are presented in fig. 8 with the flow rate $\mathrm{q}=0.2 \mathrm{~cm}^{3} / \mathrm{s}$. The different recirculation zones are found in the stenotic height for various models. We observed that the vacuum space found which have created back flow near the vessel wall after constriction zone for Newtonian and Oldroyd-B model. The parabolic profile has little deformed for non-symmetric stenosis. The comparable study of velocity and pressure for non-symmetric has shown in figs. $9 \& 10$. From the fig. 9 , the maximum velocity is found in second stenosis but lower velocity at non-symmetric stenosis.

On the other hand, the pressure distribution is presented for our considerable model with flow rate in fig. 11 There are some clearly dissimilarities among the four models at the first stenosis and second stenosis of the artery with a developed a deformed parabolic profile. Due to great shear acting on the fluid at constriction the pressure profile is more intensified. The steep pressure gradients are found far the stenosis area but in different at stenosis center. The lowest value of pressure is found in the near of second stenosis region but higher than symmetric stenosis. The most negative value is originated for generalized Oldroyd-B case at second constriction area which leads to non-Newtonian fluid.

Newtonian

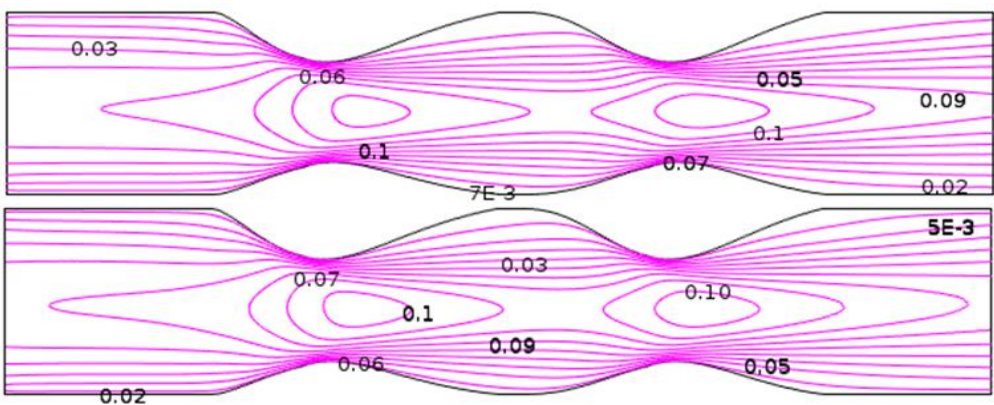


Numerical study of blood flow through symmetry and non-symmetric Stenosis artery under various ..

Oldroyd-B

Generalized

Oldroyd-B

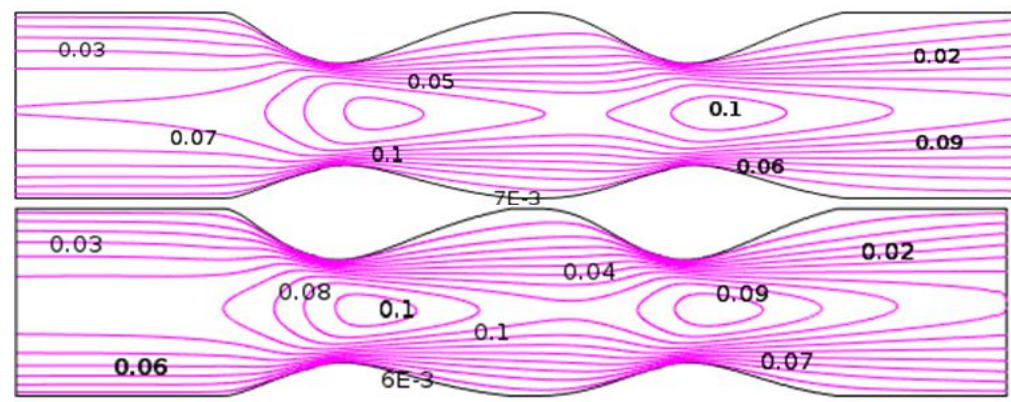

Figure 8: Velocity distribution on blood flow through non-symmetric Stenosis at $R e=100$ and $W i=0.6$

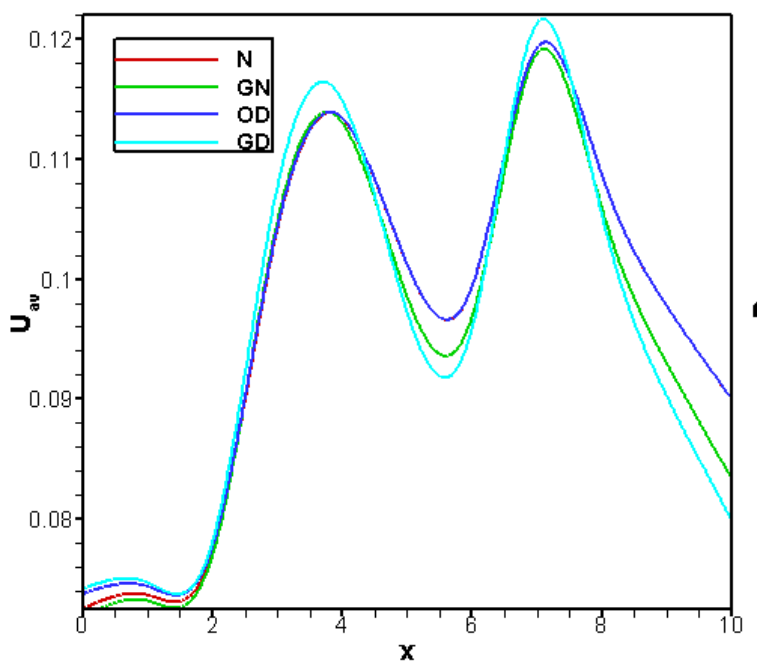

Figure 9: Effect of velocity profile of blood flow along vessel axis for non-symmetry stenosis

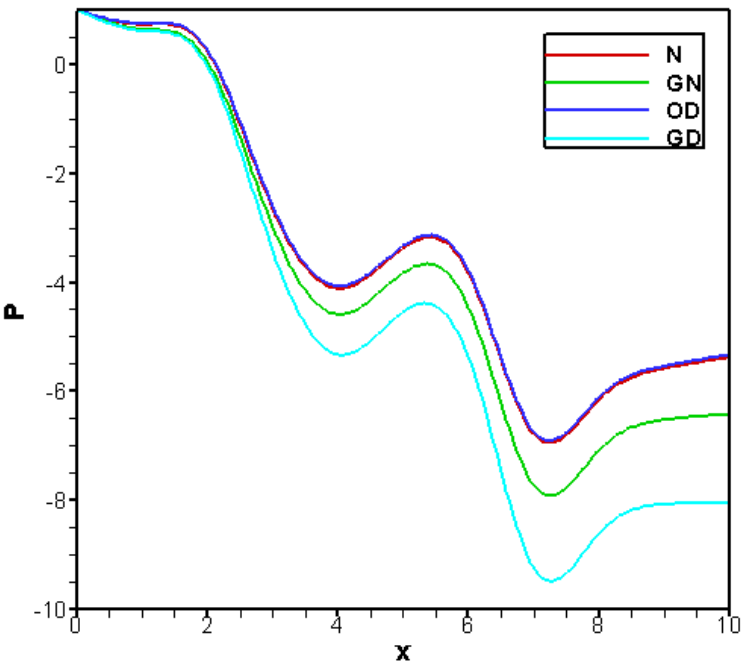

Figure 10: Effect of pressure profile of blood flow along vessel axis for nonsymmetry stenosis

Newtonian

Generalized Newtonian
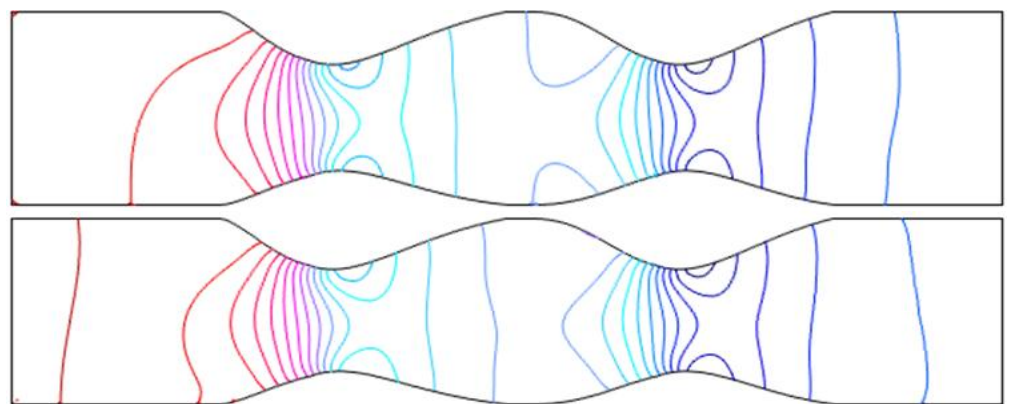

Oldroyd-B

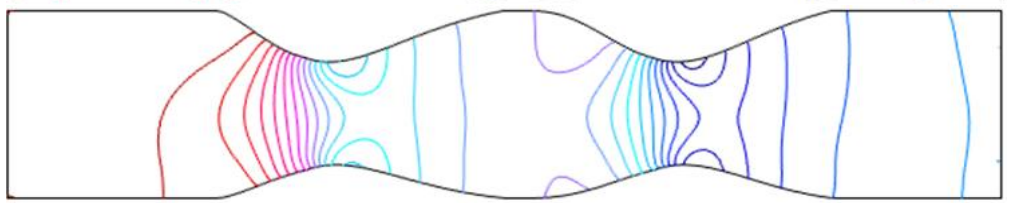

Generalized

Oldroyd-B

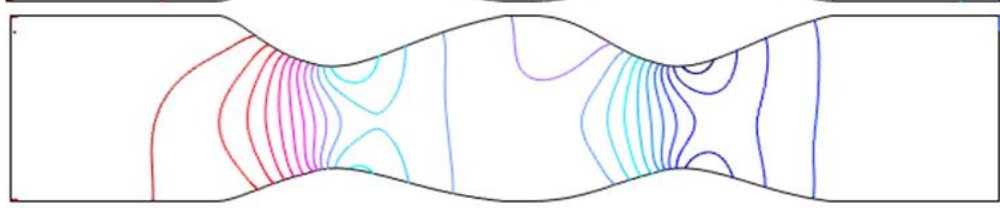

Figure 11: Pressure distribution on blood flow through non-symmetric Stenosis at $R e=100$ and $W i=0.6$ 


\subsection{Comparison between symmetric and non-symmetric stenosis}

A comparison of velocity and pressure profile are shown in figs.12-13 for Newtonian, Generalized Newtonian, Oldroyd-B, Generalized Oldroyd-B models with the flow rate $\mathrm{q}=0.2 \mathrm{~cm}^{3} / \mathrm{s}$ at $\mathrm{Re}=100$ and $\mathrm{Wi}=0.6$. The main effect of velocity and pressure are found in the constriction areas of stenosis cavity. From the figs. 12 , we observe that the velocity have a significant change at the throat of stenosis and highest value are found in second stenosis due to height of stenosis. For blood shear thinning and viscoelastic behavior the velocity is comparable lower at generalized Oldroyd-B model in the region of non-stenosis. On the other hand, the pressure have a remarkable changed at the center of stenosis are present in fig.13. The lowest value is found for all cases at symmetry stenosis because of height of stenosis. In the case of Oldroyd-B model, the pressure is more dominated at second stenosis due to high shear region.
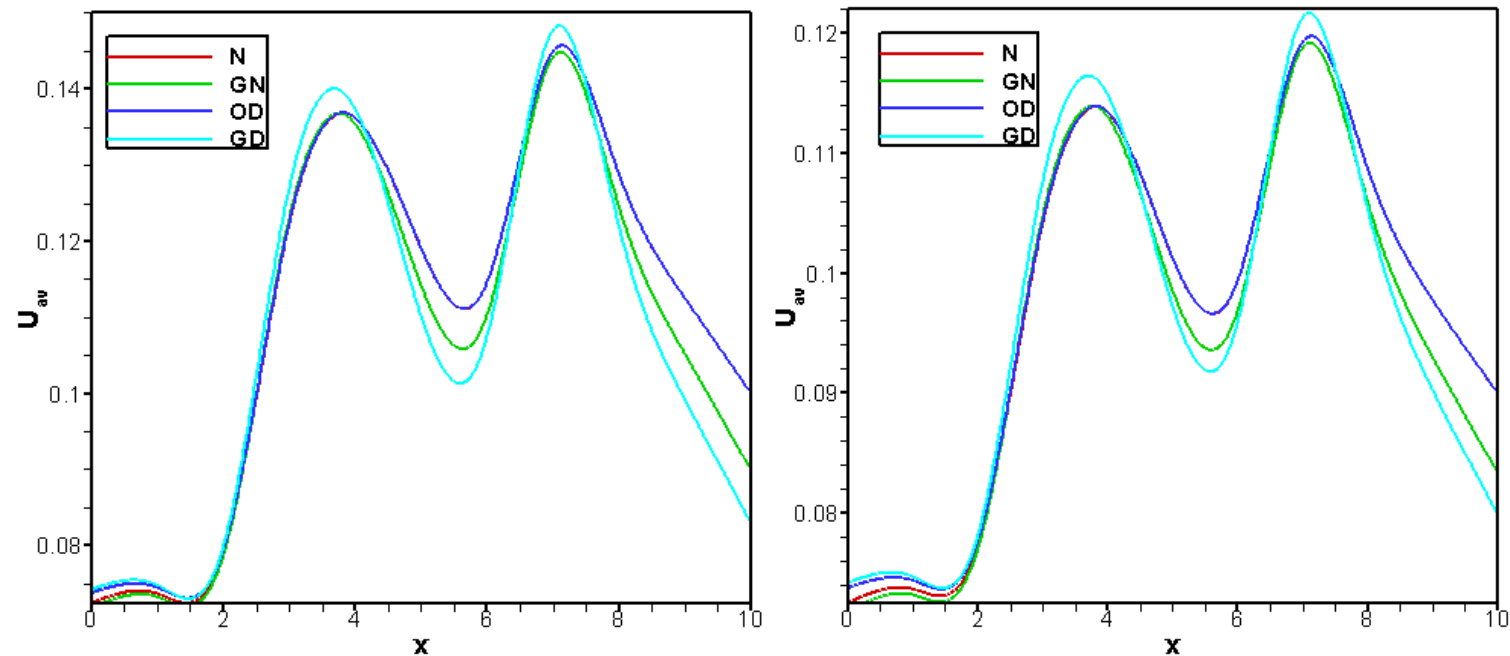

Figure 12: Comparison of Velocity profile for symmetric (left) and non-symmetric (right) stenosis effects along vessel axis
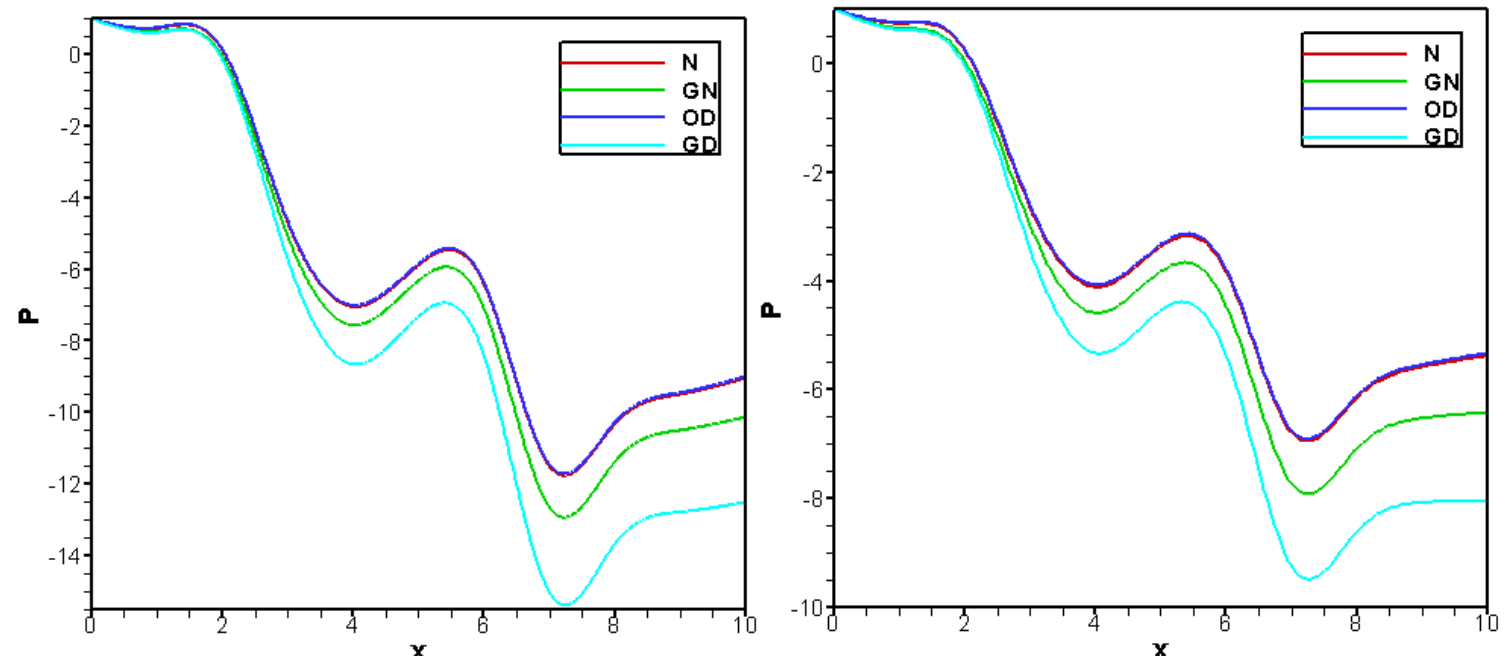

Fig 13: Comparison of Pressure profile for symmetric (Fig 3.27a) and non-symmetric (Fig 3.27b) stenosis effects along vessel axis

\subsection{Different Flow rates on Blood flow}

The axial pressure profiles are shown in figs. 14-15 at various flow rates for the four models Newtonian, Generalized Newtonian, Oldroyd-B, Generalized Oldroyd-B. By simple analysis from the figure the blood shear -thinning behavior is more intensified at stenosis area compare to non-stenosis. The velocity is little lower for generalized Oldroyd-B model compare to others. At flow rate $2 \mathrm{~cm}^{3} / \mathrm{s}$, the Newtonian and Oldroyd-B model very close and same result for rest of two models. On the other hand, the pressure distribution along vessel axis are presented in fig. 15 with different flow rates. The pressure is decreased with respect to vessel axis for all four models. At second stenosis, we obtained lowest value of pressure. For higher flow rate the pressure profile almost same for all models. 

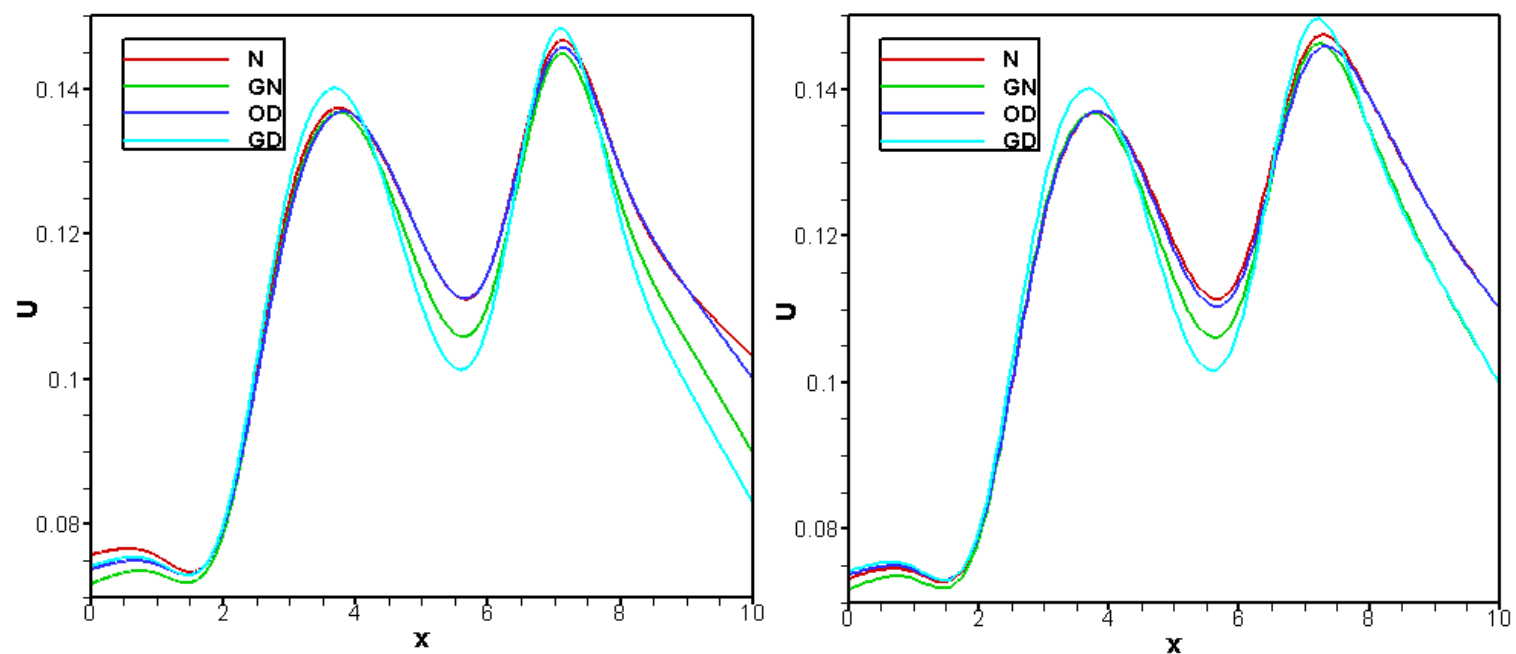

Figure 14: Velocity profile on Blood flow with various flow rate (i) $\mathrm{q}=0.05 \mathrm{~cm}^{3} / \mathrm{s}$ (left) and (ii) $\mathrm{q}=2 \mathrm{~cm}^{3} / \mathrm{s}$ (right) at $W i=0.6$ and $R e=100$
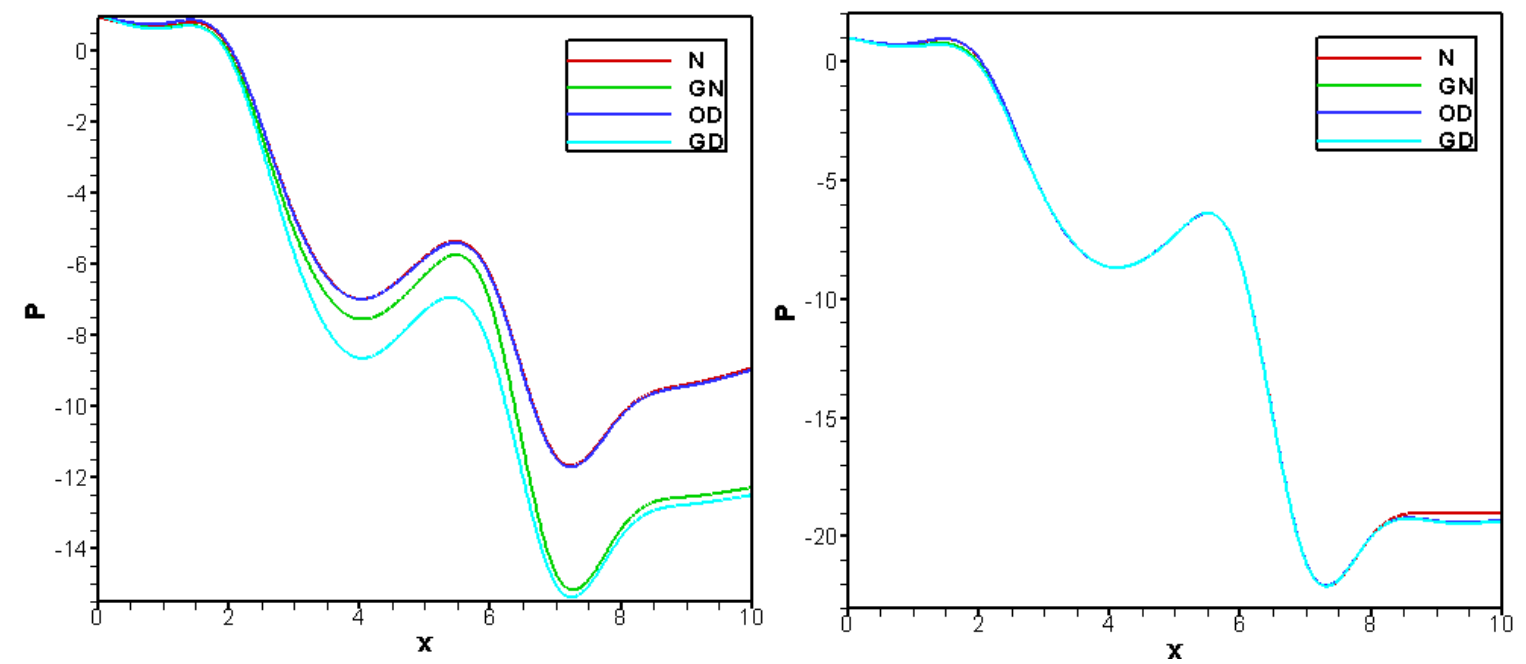

Figure 15: Pressure profile on Blood flow with various flow rate at $W i=0.6$ and $R e=100$

\section{Conclusion}

The blood flow in wavy stenosis artery is simulated by finite element method. Four constitutive laws (Newtonian model, generalized Newtonian model, Oldroyd-B models and generalized Oldroyd-B models) have been proposed to describe the non-Newtonian shear-thinning blood viscosity. The numerical investigation of Newtonian and Oldroyd-B models, and their generalized (shear-thinning) models have been considered to the blood flow through symmetric and non-symmetric stenosis in steady flow simulations. From the computational results, we conclude that the effect of flow variables and wall shear stress are related to viscoelasticity (shearthinning) are more noticeable than the viscoelastic ones. At the throat of stenosis, the flow variables are predominant and increased with the flow rate decreased consequently. The specific choice of the characteristic viscosity $\mu_{\mathrm{n}}$ for the reference Newtonian and (non-generalized) Oldroyd-B solution is main reason. The apparent viscosity is close to $\mu_{0}$ in the large part of vessel at low shear rates and the difference for the Newtonian solution at high flow rate. For the given problems, the numerical method used to solve the governing equations seems to be sufficiently robust and efficient for the appropriate resolution.

The finite element equations derived from the governing flow equations that consist of the conservation of mass, momentum, and Oldroyd-B equations. The derived finite element equations are nonlinear requiring an iterative technique solver. The Newton-Raphson iteration method has been applied to solve these nonlinear equations for solutions of the nodal velocity component, tensor component, and pressure by considering Weissenberg numbers of 0.0, 0.5 and 1.00 and Reynold numbers of $10^{2}$ to $10^{3}$. The results show the following aspects:

(i) The effect of flow variables (velocity and Pressure) have more significant changed at the throat of stenosis for all four models. 
(ii) The effect of the blood flow behavior on the wall shear stress is an important factor in the onset of arterial diseases.

(iii) The Peak value of blood velocity and lowest value of blood pressure are found at second constriction region for our considerable models.

(iv) It is another remarkable effect on blood flow if the height of stenosis is different of the four dissimilar models.

(v) Various types of recirculation zone are originated at the throat of stenosis regions for all four models.

\section{Acknowledgements}

Authors would like to thank the Department of Information and Communication Technology (ICT), Bangladesh University of Professionals (BUP), for providing computer and others facilities during this work. We also express our gratitude to anonymous reviewers for their suggestions which improved the quality of the manuscript.

\section{References}

[1] Anand, M., Rajagopal, K. R., "A shear-thinning viscoelastic fluid model for describing the flow of blood”, International Journal of Cardiovascular Medicine and Science, Vol. 4 (2), 2004, pp.59-68.

2] COMSOL Multiphysics, 4.3a users guide, 2013

[3] Dechaumphai, P., Adaptive finite element technique for heat transfer problems, Energy, Heat \& Mass transfer, 17(2) .1995, 87-94.

[4] Dechaumphai, P., Finite Element Method in Engineering (Bangkok, Chulalongkorn University Press, 1999)

[5] D' Elia, M., Perego, M., Veneziani A., "A variational Data Assimilation procedure for the incompressible Navier-Stokes equations in hemodynamics”, J. Sci. Comput., Vol. 52(2), 2011, pp. 340-359.

[6] Kumar, B. R., Kumar, G.A., Kumar, S.M., "MATLAB ${ }^{\mathrm{R}}$ and its Application in Engineering" (Panjab University, India, 2010).

[7] Lowe D., "Clinical Blood Rheology", (CRC Press, Boca Raton, Florida, 1998).

[8] Marshall, I., Zhao, S., Sopoulou P. P, Hoskins, P., and Xu, X. Y., "MRI and CFD studies of pulsatile flow in healthy and stenosed carotid bifurcation models," Journal of Biomechanics, Vol. 37(5), 2004, pp. 679-687.

[9] Oka, S., "Pressure development in a non-Newtonian flow through a tapered tube," Biorheology, Vol. 10(2), 1973, pp. 207-212.

[10] Prokop, V. and Kozel, K, "Numerical simulation of Generalized Newtonian and Oldroyd-B Fluids", Numerical Mathematics and Advanced Application 2011, 2013, pp 579-586,

[11] Keslerova, R., Karel, K., "Numerical modelling of viscous and viscoelastic fluids flow through the branching channel", Programs and Algorithms of Numerical Mathematics, Institute of Mathematics AS CR, Prague 2015, pp. 100-105

[12] Mahfoud, M., Benhadid S., Numerical study of the non-Newtonian blood flow in a stenosed artery using two rheological models, Thermal Science, Vol. 20, No. 2, 2016, pp. 449-460

[13] Rajagopal, K.R., Srinivasa, A.R., "A Gibbs-potential-based formulation for obtaining the response functions for a class of viscoelastic materials", Proc. R. Soc. A, Vol. 467, 2011, pp 39-58,

[14] Robertson, A. M., Sequeira, A., Owens, R. G., "Rheological models for blood", in: Formaggia, L., Quarteroni, A., Veneziani, A. (Eds.), "Cardiovascular Mathematics, Modeling and simulation of the circulatory system" MS\&A, Modeling, Simulations \& Applications, Vol. 1, 2009, pp. 211-241.

[15] Taylor,C., Hood,P., A Numerical Solution of the Navier-Stokes Equations Using Finite Element technique, Computer and Fluids 1, 73, 1973. doi:10.1016/0045-7930(73)90027-3

[16] Telma, G., Jorge, T., Adelia, S., "Optimal control in blood flow simulations”, Int. Journal of Non-Linear Mechanics, Vol. 64,2 014, 57-69.

[17] Thurston, G.B., "Frequency and shear rate dependence of viscoelasticity of blood", Biorheology, Vol.10(3), 1973, 375-381.

[18] Tu, C. and Deville, M., "Pulsatile flow of non-Newtonian fluids through arterial stenoses," Journal of Biomechanics, Vol. 29(7), 1996, pp. 899-908.

[19] Verdier, C., "Rheological properties of living materials. from cells to tissues", Journal of Theoretical Medicine, Vol. 5 (2), 2003, 67-91. 